\title{
Realce textural mediante "desbaste iónico" de muestras pulidas de clínker de cemento portland para estudios por microscopía electrónica de "scanning"
}

\author{
ANGel RODRIGUEZ REY (Dpto. de Petrología. Ftad. de Geología. Universidad de Oviedo) \\ CARLOS DE LLANOS GONZALEZ (Servicio de Microscopia Electrónica de "scanning". Universidad de Oviedo)
}

\section{$R E S U M E N$}

Se estudian los resultados de la aplicación del "desbaste iónico" como etapa final de la preparación de muestras pulidas de clinker de cemento portland para estudios por microscopia electrónica de "scanning". Se observa que, bajo condiciones apropiadas, se obtienen superficies planas y limpias que permiten distinguir rasgos texturales (tamaño, forma y bordes de grano) que, dadas las similares características de emisión de electrones secundarios que poseen los minerales del clínker, son de dificil discriminacion en secciones pulidas convencionales.

\section{$S U M M A R Y$}

Results of using "ion thinning" techniques as final stage in the preparation of polished samples of portland cement clinker for SEM studies are showed. Under proper conditions, flat and clean surfaces are obtained which allow to discriminate textural facts (grain size, form, etc.), hardly to see on conventional polished samples due to the similarities of the secondary electron emission of the clinker minerals.

\section{INTRODUCCION}

La combinación de la microscopía electrónica de "scanning" con un sistema de análisis de rayos $\mathrm{X}$ acoplado al microscopio, constituye una técnica muy útil y completa para el estudio del clínker de cemento portland. De esta forma se pueden obtener datos cristalográficos, químicos y morfológicos (texturales) de una misma zona de la muestra.

El tipo de muestra y su preparación adquieren, en este caso, gran importancia. Las muestras de superficie de fractura, aunque fáciles y rápidas de preparar, no son las más adecuadas para ciertos tipos de estudios mediante SEM: cuantificación de fases, observación y cuantificación de porosidad, confección de mapas composicionales o de distribución de elementos en el interior de las fases mediante análisis de rayos $\mathrm{X}$, etc.

Según Beaman e Isasi (1), las muestras idóneas para estudios mediante microsonda electrónica deben poseer ciertas características:

- superficies planas y limpias;

- rasgos morfológicos fáciles y rápidos de identificar.

Por lo tanto, es más conveniente la utilización de muestras pulidas de clínker preparadas siguiendo cualquiera de los métodos ya habituales. 
Sin embargo, las distintas fases mineralógicas del clínker poseen una emisión de electrones secundarios muy similar que dificulta la fácil y rápida discriminación de las características texturales de la muestra. Es por ello que se hace necesario el empleo de técnicas de realce textural.

Barnes et al. (2), señalan algunas posibles técnicas aplicables a este caso:

- obtención de imágenes con electrones primarios retrodispersados;

- mapeo mediante análisis de rayos $\mathrm{X}$;

- ataque superficial con reactivos químicos;

- desbaste iónico.

En su opinión, el procedimiento más adecuado es el desbaste iónico: las imágenes obtenidas con electrones retrodispersados no siempre discriminan lo suficiente; el mapeo mediante análisis de rayos $\mathrm{X}$ no siempre es práctico, y el ataque superficial con reactivos químicos tiene los inconvenientes de que se pueden formar depósitos de productos de reacción sobre la superficie además de la posible lixiviación de elementos de la muestra durante el ataque.

La técnica del desbaste iónico, aunque ya conocida desde la década de los 50, no se ha empezado a utilizar hasta hace pocos años como método de preparación de muestras de materiales inorgánicos muy diversos para microscopía electrónica (3) (4) (5). Además de su aplicación a la preparación de superficies para SEM, también se aplica para obtener láminas ultradelgadas ("thin-foils") para microscopía electrónica de transmisión (TEM).

Los primeros trabajos sobre preparación de superficies de clínker para SEM mediante desbaste iónico, fueron realizados por Barnes et al. (2). Otros autores (6) (7), han utilizado esta técnica para la obtención de "thin-foils" de clinker para TEM.

En el presente trabajo sólo se trata de su aplicación a la preparación de superficies para SEM.

En el siguiente apartado se describe, de forma general, el equipo instrumental y los fundamentos de esta técnica.

\section{DESCRIPCION DE LA TECNICA}

El desbaste iónico es el proceso de eliminación de material de una superficie llevado a cabo por la acción de iones acelerados de elevada energía. Es, pues, un proceso de "sputtering". Generalmente se utilizan iones de argón con energía variable entre 1 y $10 \mathrm{keV}$. Cada uno de ellos al incidir sobre la muestra expulsa unos pocos átomos de la superficie bombardeada (típicamente 2 ó 3 átomos en el caso de iones de $5 \mathrm{keV}$ ). Es, pues, una extracción de material átomo a átomo, lo cual implica un daño mínimo a la estructura original de la muestra.

Según la cantidad de material eliminado, Barnes et al. (2), distinguen entre "ion beam etching" y "ion milling"; el primer término se utiliza para el caso de erosión ligera o diferencial, mientras que el segundo implica una extracción de material más significativa (superior a las $2 \mu \mathrm{m}$ de profundidad, más o menos).

El rendimiento de un proceso de desbaste iónico se define como el número de átomos de la muestra bombardeada que son expulsados por cada ión incidente. La velocidad de desbaste es el espesor de la capa superficial erosionada por unidad de tiempo. Dada la naturaleza del proceso, la velocidad de desbaste iónico es muy pequeña; en el caso del clínker de cemento portland varia entre 0.1 y $1 \mu \mathrm{m}$ por hora dependiendo de las caracteristicas especificas del aparato (2). 
La velocidad de desbaste es, lógicamente, una medida de su rendimiento.

Tal como cabría esperar, cada material específico tiene una velocidad de desbaste particular distinta de la de los demás materiales, puesto que en ella influyen tanto la relación másica entre el ión incidente y el átomo bombardeado, como la estructura cristalina del material. Además, para un mismo cristal de un material, la velocidad de desbaste es diferente en cada una de las distintas secciones de distinta orientación que pueden obtenerse de él, al variar, entonces, la densidad atómica.

La erosión diferencial resultante permite la utilización del desbaste iónico como método de realce textural: se pueden diferenciar, entonces, características texturales (distribución y tamaño de grano de las fases, bordes de grano...), tanto en superficies monofásicas policristalinas como en superficies polifásicas compuestas por minerales con similar emisión de electrones secundarios y, por tanto, de difícil discriminación bajo el microscopio electrónico de "scanning".

Por otra parte, el rendimiento del desbaste de cada material específico varía de acuerdo con las condiciones del proceso de bombardeo. Tal como se recoge en (4), los principales parámetros que controlan este rendimiento son:

- la energía de los iones incidentes;

- el ángulo de incidencia que forma el haz de iones con la superficie de la muestra.

Cuanto mayor es la energía de los iones incidentes, más elevada resulta la velocidad de desbaste. Sin embargo, también aumenta el riesgo de dañar la estructura original de la muestra.

El rendimiento del desbaste aumenta conjuntamente con el ángulo de incidencia del haz, alcanzándose el máximo con ángulos de $30^{\circ}$. Por encima de este valor el rendimiento disminuye, debido a que los iones inciden demasiado perpendicularmente y utilizan su energía para profundizar más en el interior de la muestra, pero sin expulsar átomos de su superficie.

Para el presente trabajo se ha utilizado una estación de bombardeo iónico POLARON, del Departamento de Petrología de la Universidad de Oviedo. En la foto 1 se muestra una imagen

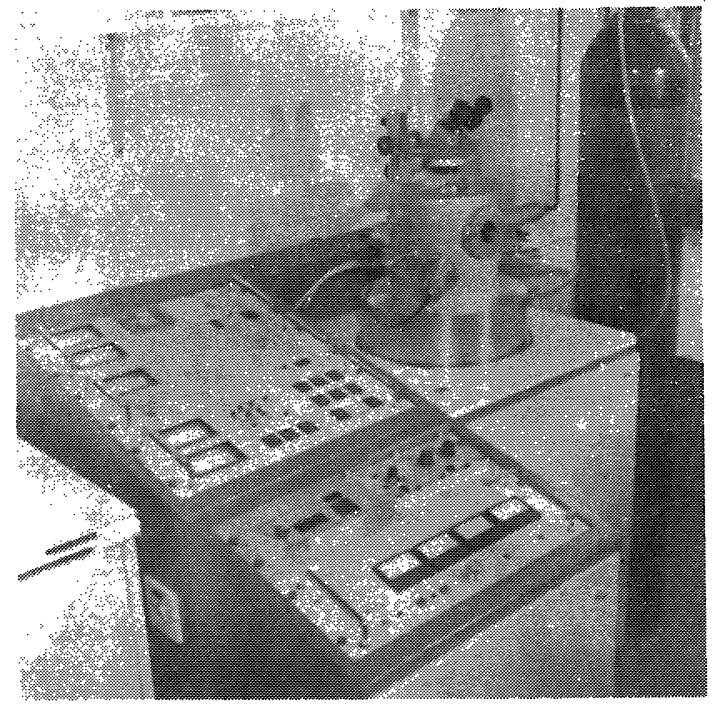

a)

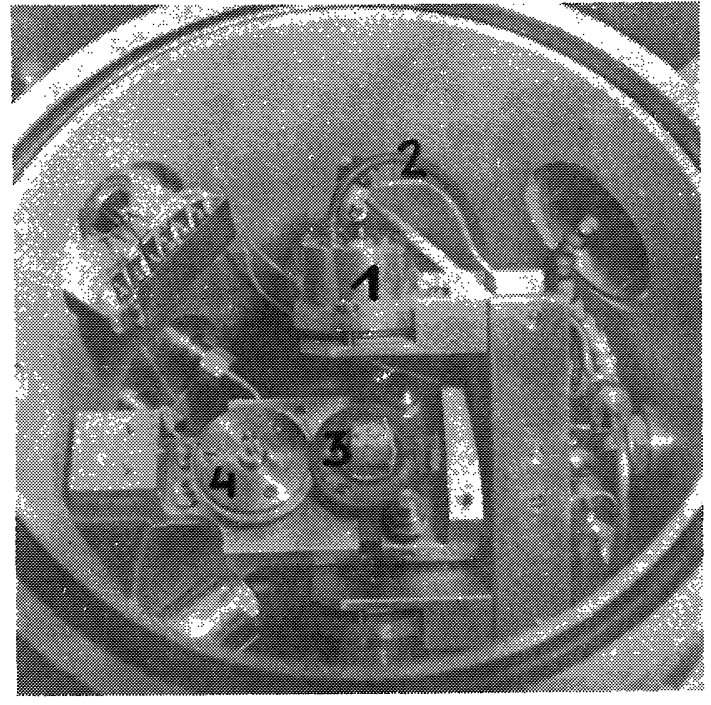

b)

Foto 1a).-Fstación de desbaste iónico POLARON utilizada en este trabajo. 1b). Detalle del interior de la cámara de desbaste: 1. cañon donde se produce el haz de iones de argon: 2, conducto de entrada de gas al cañon: 3. muestra: 4. dispositivo de giro de la muestra. 
del equipo completo, así como la configuración detallada de la cámara de desbaste. El bombardeo de la muestra se efectúa dentro de esta cámara una vez conseguido un vacio mejor que $10^{-5}$ torr.

Cuando se preparan muestras para microscopia electrónica de transmisión, se utilizan dos cañones simétricos y opuestos respecto a la lámina ultradelgada a obtener, los cuales la bombardean simultáneamente mediante dos haces cilindricos de unos $2 \mathrm{~mm}$ de diámetro. En nuestro caso, dado que la observación iba a llevarse a cabo mediante SEM, se utilizó un solo cañón que lanzaba un haz más grande, de $10 \mathrm{~mm}$ de ancho, lo que permitía desbastar superficies mayores (típicamente $1 \mathrm{~cm}^{2}$ si la distancia del cañon a la muestra es de $5 \mathrm{~cm}$ ). La muestra puede hacerse girar, mientras es desbastada, alrededor de un eje perpendicular a su superficie, a velocidad constante y regulable entre 0.3 y 10 r.p.m. Los cañones pueden hacerse bascular para conseguir que el ángulo de incidencia de los haces adquiera valores comprendidos entre 0 y $90^{\circ}$.

Como partículas incidentes se utilizaron iones de argón, tal como es habitual en la mayoría de trabajos, ya que no interaccionan quimicamente con la muestra, y están disponibles en forma atómica como gas relativamente pesado.

Antes de ser sometido a desbaste iónico, la muestra ha de ser convenientemente pulida utilizando, en la última etapa del proceso, pasta de diamante o alúmina de $0.25 \mu \mathrm{m}$ de tamaño, como mínimo. Después se pega al portamuestras en el que se va a introducir en el microscopio de "scanning" y se coloca dentro de la cámara de desbaste.

Una vez alcanzado, dentro de la cámara, un vacio de $10^{-5}$ torr, puede iniciarse el proceso de desbaste.

Después del periodo de desbaste, la muestra se recubre con una capa fina de Au-Pd (20 $\mu \mathrm{m})$ en una metalizadora por "sputtering", quedando dispuesta para su observación mediante microscopía electrónica de "scanning".

\section{PROCESO EXPERIMENTAL}

Ya se ha mencionado que en los equipos comerciales pueden seleccionarse varios parámetros (energía de los iones, ángulo de incidencia, etc.) que influyen en el rendimiento y velocidad del desbaste. Es importante, entonces, elegir las condiciones más apropiadas para conseguir el resultado deseado.

Por otra parte, bajo determinadas condiciones el proceso de desbaste iónico puede introducir modificaciones morfológicas ("artefacts") en la superficie de la muestra cuya aparición hay que evitar. La localización de los "artefacts" está condicionada por la presencia de defectos cristalinos en las zonas superficiales de la muestra. Su evolución puede predecirse según la teoría cinemática de Frank sobre la disolución de los cristales en función de su orientación cristalográfica (8).

En general, los "artefacts" pueden clasificarse morfológicamente en dos tipos: microcráteres y microondulaciones. El que aparezca uno u otro tipo depende, en gran parte, de ciertas condiciones del desbaste: ángulo de incidencia y velocidad de giro de la muestra.

Barnes et al. (2), han estudiado la relación entre estos parámetros y los "artefacts" desarrollados en secciones pulidas de clínker sometidas a desbaste iónico. Al igual que otros autores, encontraron que con ángulos de incidencia de $90^{\circ}$, aparecían gran cantidad de microcráteres que aumentaban gradualmente de tamaño a medida que se incrementaba la duración del desbaste. En 
muestras que se mantenían en rotación, y con ángulos de incidencia mucho menores (entre $5 \mathrm{y}$ $20^{\circ}$ ), no se desarrollaban "artefacts" durante las primeras etapas del proceso ( 2 a 3 horas), siempre que la energía de los iones de argón no fuese excesivamente elevada (un valor apropiado sería de $5 \mathrm{keV}$ ). Sin embargo, al aumentar la duración del proceso, comenzaba la aparición paulatina de "artefacts" superficiales en forma de micro-ondulaciones.

Teniendo en cuenta las recomendaciones de dichos autores, se ha procedido al desbaste iónico de varias muestras pulidas de cemento portland, obtenidas siguiendo un método habitual de preparación (9).

Para cada muestra se ha modificado alguno de los parámetros del proceso de desbaste para mostrar su influencia en el resultado final y establecer, entonces, las condiciones más apropiadas.

Así, en las fotos $2 \mathrm{a}$ y $2 \mathrm{~b}$, puede seguirse la evolución de la misma zona de una de las muestras sometida a desbaste por impacto de iones de argón de $5 \mathrm{keV}$ de energía bajo un ángulo de incidencia de $20^{\circ}$, y que giraba a 2 r.p.m. Antes del proceso (foto $2 \mathrm{a}$ ), la discriminación de los rasgos texturales, especialmente los contornos y formas de los granos, es prácticamente imposible. $\mathrm{La}$ foto $2 \mathrm{~b}$ muestra su aspecto después de 3 horas de desbaste. El espesor de la capa desbastada es, en estas condiciones, de $0,3 \mu \mathrm{m}$ aproximadamente. Las características texturales son ahora fácil y rápidamente discriminables. El realce textural se debe a la erosión diferencial ya mencionada; los fenocristales de alita y de belita, son erosionados más rápidamente que las fases intersticiales; el bajo ángulo de incidencia hace que el relieve se mantenga constante $\mathrm{y}$ dentro de limites aceptables debido al efecto de apantallamiento. Los mismos buenos resultados pueden observarse en otra zona de la misma muestra fotografiada a mayores aumentos, fotos $3 \mathrm{a}$ y $3 \mathrm{~b}$. Si se aumentase el tiempo de desbaste por encima de $10 \mathrm{~h}$, irian apareciendo "artefacts" en forma de micro-ondulaciones, que, al final, enmascararian las características texturales originales de la muestra. Estas son, pues, las condiciones de bombardeo ideales (velocidad de giro, ángulo de incidencia, y tiempo de desbaste) para conseguir una superficie óptima.

Si se mantiene la muestra inmóvil, sin modificar las restantes condiciones de bombardeo, el resultado final, aunque aceptable, no es tan bueno como en el caso anterior, fotos 4 (a, b, y c). El apantallamiento sólo se produce en los bordes dispuestos perpendicularmente y del lado contrario con respecto a la dirección de ataque, y, en consecuencia, el realce textural no es tan bueno como antes.

El ángulo de incidencia del haz tiene bastante importancia en los resultados que se obtienen. En

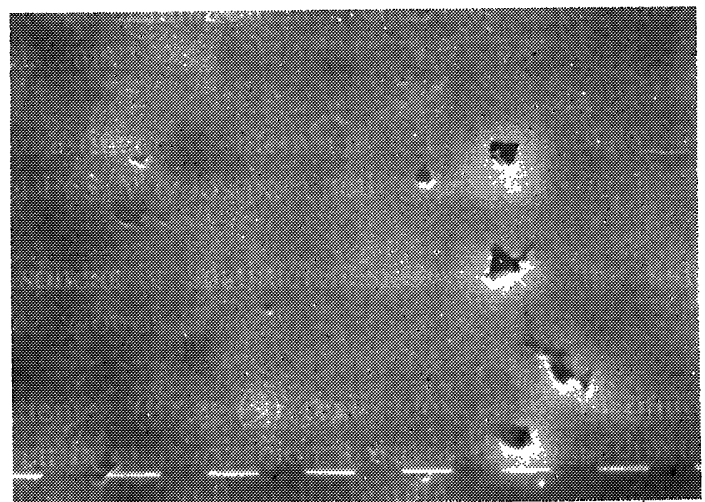

a)

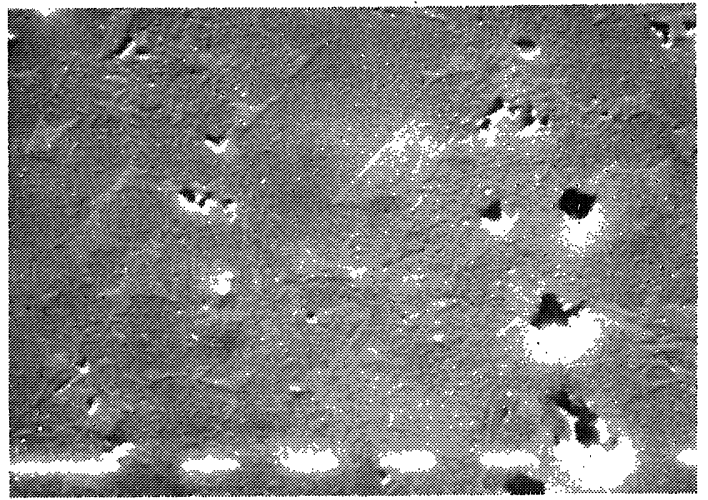

b)

Fotos 2a y 2b.--Muestra sometida a desbaste en las siguientes condiciones: tiempo, 3 h.: energía del haz. 5 keV: angulo de incidencia, 20\% velocidad de giro de la muestra, 2 r.p.m. a) antes del proceso. b) superficic resultante. ( $\times 750$. 1 marcador $=10 \mu \mathrm{m})$. 
las fotografías $5 \mathrm{a}$ y $5 \mathrm{~b}$, se muestran los efectos del bombardeo efectuado con un ángulo de incidencia de $90^{\circ}$, manteniendo inmóvil la muestra. Estas condiciones dan lugar a la aparición de numerosos "artefacts" tipo micro-cráteres que irían aumentando en tamaño y número a medida que progresase el desbaste. No ha habido efecto de apantallamiento y el realce textural no ha sido muy bueno. La superficie resultante no es la más apropiada para el estudio combinado por microscopía electrónica de "scanning" y análisis de rayos $\mathrm{X}$.

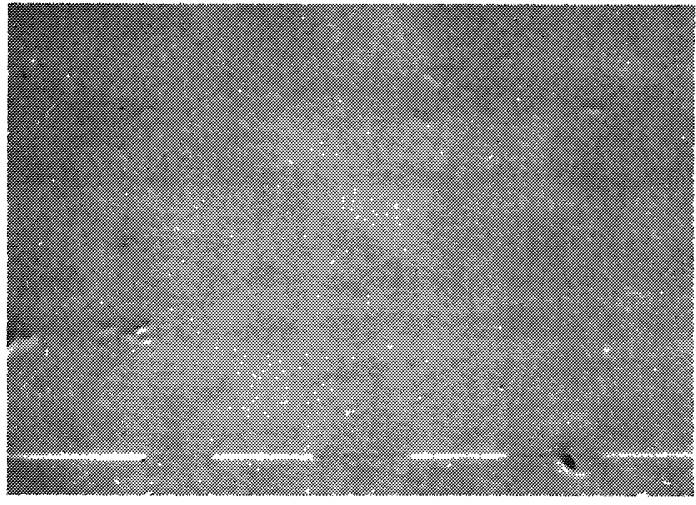

a)

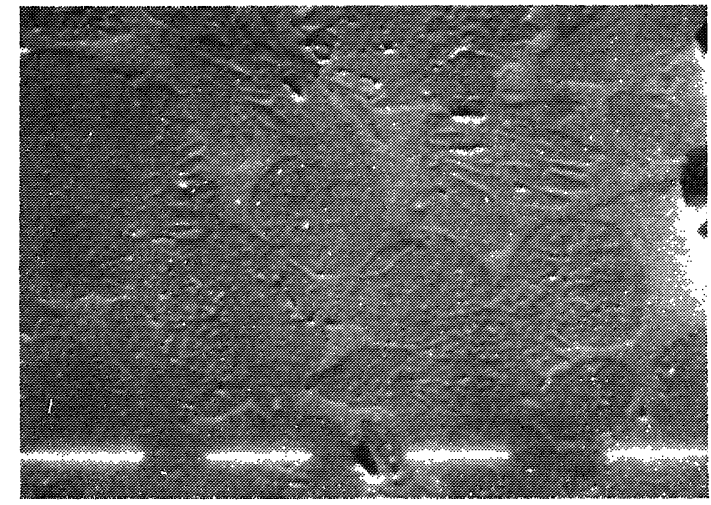

b)

Fotos 3a y 3b.-Otra zona de la misma muestra de la foto anterior observada a mayores aumentos. a) antes del desbaste. b) superficie resultante; el realce textural obtenido es muy bueno: los cristales de alita y de belita aparecen en bajorrelieve debido a su mayor velocidad de desbaste; se distinguen perfectamente los contornos de las belitas esqueléticas. $(\times 1.450$, 1 marcador $=10 \mu \mathrm{m})$.

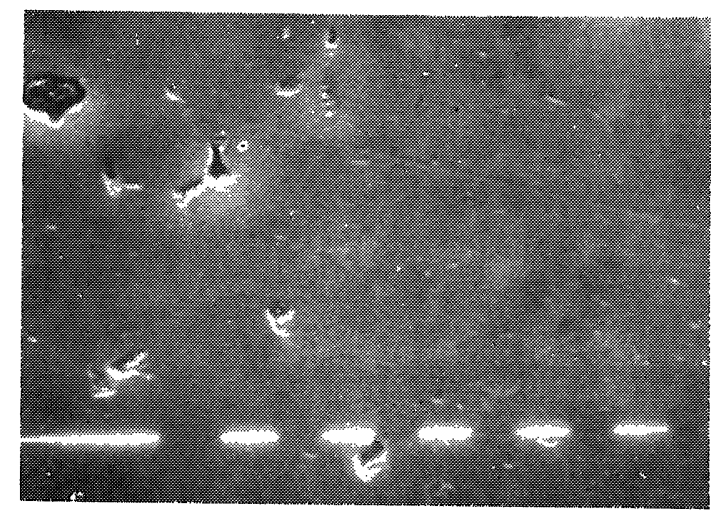

a)

Fotos $4 \mathrm{a}$. 4b y $4 \mathrm{c}$.- Muestra sometida a desbaste iónico durante $3 \mathrm{~h}$ con iones de $5 \mathrm{keV}$ bajo un ángulo de $20^{\circ}$. mantenida inmóvil durante ci proceso. a) antes del desbaste. b) superficie resultante. c) detalle de la superficie desbastada: la dirección del haz ha sido N-S: obsérvese como la matriz. que resalta sobre los fenocristales, presenta un espesor en cuña con las "vertientes meridionales" más abruptas. (a y b, $\times 750$ : c. $\times 1.450$; en todas ellas 1 marcador $=10 \mu \mathrm{m}$ ).

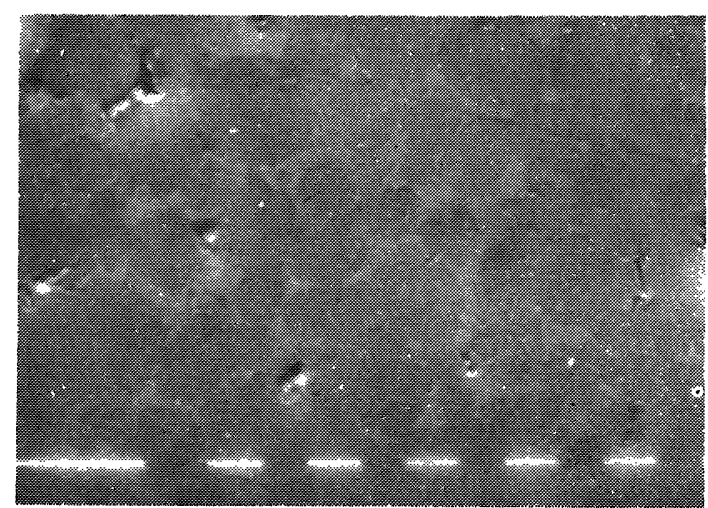

b)

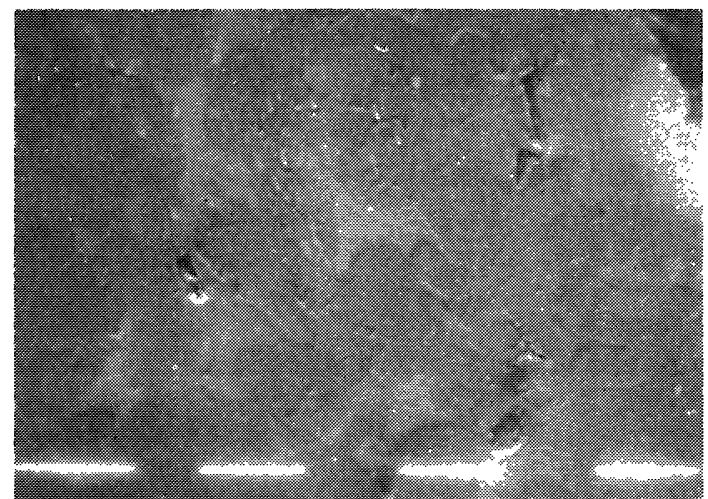

c) 


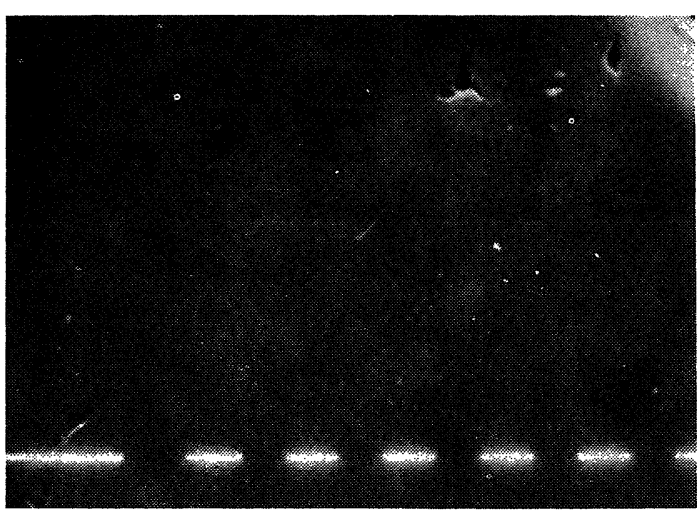

a)

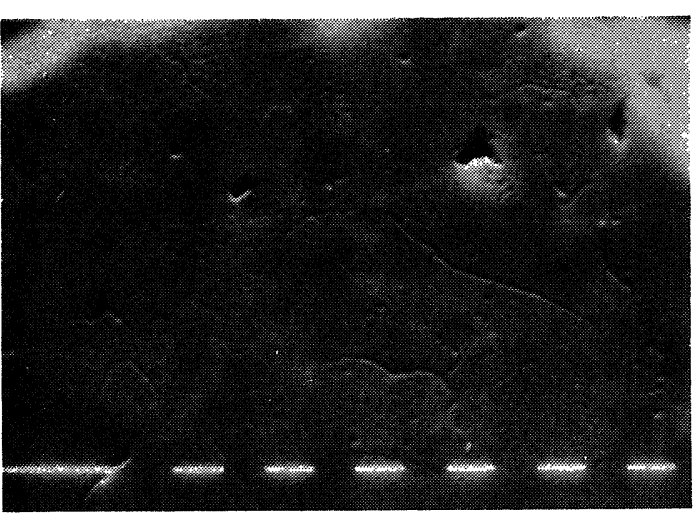

b)

Fotos 5 a y $5 \mathrm{~b}$.-Muestra sometida a $3 \mathrm{~h}$ de desbaste, sin girar, con iones de $5 \mathrm{keV}$ que incidian perpendicularmente. a) antes del desbaste. b) resultado del proceso; obsérvese la aparición de "artefacts" en forma de microcráteres. ( $X 750,1$ marcador $=10 \mu \mathrm{m})$.

\section{CONCLUSIONES}

Además de permitir la obtención de láminas ultradelgadas ("thin-foils") para microscopía electrónica de transmisión, el desbaste iónico es muy útil como etapa final de la preparación de muestras de clínker de cemento portland para estudios combinados de microscopía electrónica de "scanning" y análisis de rayos $\mathrm{X}$.

Con su uso se obtienen superficies planas y limpias en las que pueden distinguirse características texturales que de otro modo resultarían difícilmente observables. El realce textural de estas muestras permite llevar a cabo su estudio más fácil y rápidamente.

La introducción de características morfológicas no originales de la muestra ("artefacts") por acción del proceso de bombardeo, puede minimizarse si se disponen las condiciones apropiadas: ángulos de incidencia pequeños $\left(0-25^{\circ}\right)$, energia de los iones de argón no superior a $5 \mathrm{keV}$, y muestra en rotación. Los resultados de este trabajo confirman los estudios sobre las condiciones idóneas recomendadas por otros autores.

El empleo de esta técnica es especialmente apropiado, también, cuando vayan a realizarse determinaciones cuantitativas texturales (determinación de perimetros y áreas de fases minerales) en imágenes de microscopía electrónica de "scanning", mediante procedimientos automatizados (proceso digital de imágenes).

\section{BIBLIOGRAFIA}

(1) BEAMAN, D. R. e ISASI, J. A.: (1971). "Electron beam microanalysis". Mater. Res. \& Stand., V. 11, pp. 11-56. A.S.T.M.

(2) BARNES, P.; FONSEKA, G. M.; GHOSE, A.; MOORE, T.: (1979). "Ion beam etching in the study of cementitious materials". J. Mater. Sci., V. 14, pp. 2.831-2.836.

(3) BARBER, D. J.: (1970). "Thin foils of non-metals made for electron microscopy by sputter-etching". J. Mater. Sci., V. 5 , pp. $1-8$.

(4) GOODHEW, P. J.: (1972). "Specimen preparation in Materials Science". En: Practical methods in electron microscopy. A. M. Glauert, Editor. North Holland Publ. Co. Amsterdam.

(5) RODRIGUEZ REY, A. y MONTOTO, M.: (1984). "Aplicación del «desbaste iónico» en la preparación de muestras rocosas para su estudio textural mediante SEM". Comunicaciones del I Congreso Español de Geologia. T. III, pp. 469-476. 
(6) GROVES, G. W.: (1981). "Portland cement clinker wiewed by transmission electron microscopy". J. Mater. Sci., V. 16 , pp. $1.063-1.070$.

(7) GHOSE, A. y BARNES, P.: (1981). "Microstructural investigations on Portland cement clinkers by ion beam etching and thinning techniques". Indian Concr. J., V. 9, pp. 255-258.

(8) BARBER, D. J.; FRANK, F. C.; MOSS, M.; STEEDS, J. W. \& TSONG, I. S. T.: (1973). "Prediction of ionbombarded surface topographies using Frank's Kinematic theory of crystal dissolution" J. Mater. Sci., V. 8, pp. 1.0301.040 .

(9) FERNANDEZ PARIS, J. M.: (1968). "Microscopía del clínker de cemento Portland". Inst. Eduardo Torroja de la Construcción y del Cemento. Madrid.

\section{AGRADECIMIENTOS}

Los autores desean expresar su agradecimiento a D. Enrique Fernández Molina y demás miembros del laboratorio Estabisol, por las facilidades prestadas para la realización de este trabajo.

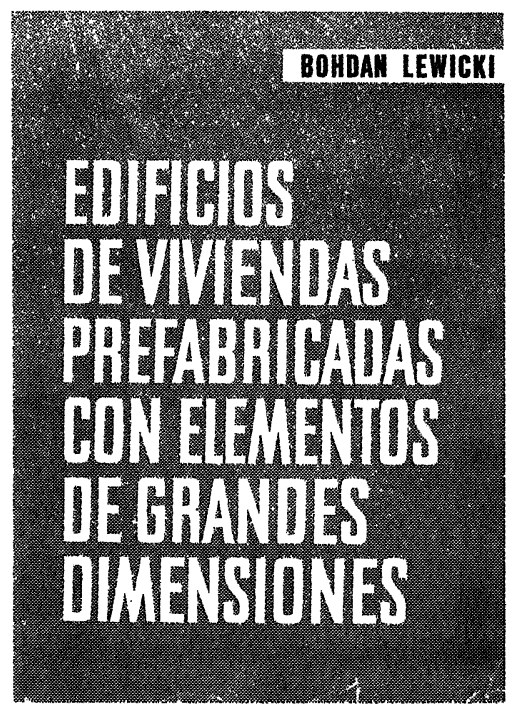

Bohdan Lewicki

Este libro trata de los problemas relativos a la construcción de los edificios de viviendas o publicos realizados con elementos prefabricados de grandes dimensiones. Se han estudiado los problemas de arriostramiento, asi como los que plantea la resistencia de los elementos y de la estructura; se han los elementos y de la estructura; se han
examinado las cuestiones de orden higrotérmico, acústico y de resistencia al fuego: también se ha profundizado en el estudio de la estanquidad de los muros exteriores y de las juntas.

La obra incluye numerosas ilustraciones que dan detalles de diversas soluciones, as como ejemplos de cálculo, tablas de valores numéricos, diagramas y ábacos.

Un volumen encuadernado en tela, de $24 \times 17 \mathrm{~cm}$, compuesto de 616 págs.

Precios: 2.500 ptas.; \$USA 36.00 .

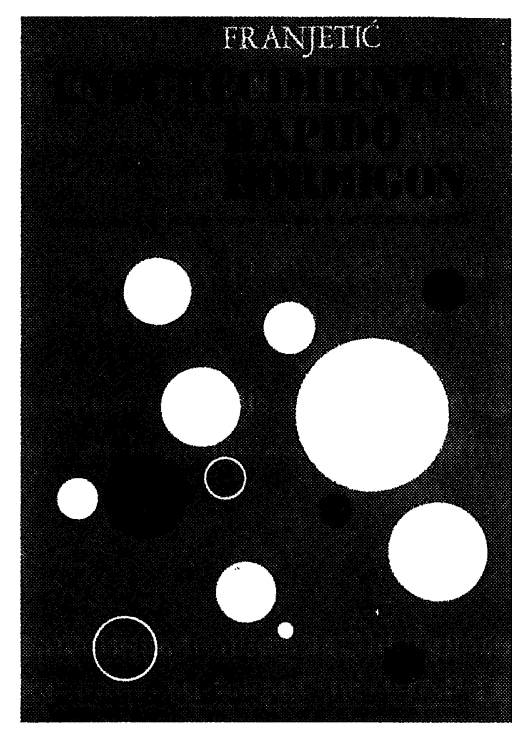

\section{Zorislav Franjetic}

En la obra de Franjetic se expone de una forma minuciosa, ordenada y sistemática, todo un cuerpo de doctrina que reúne el conocimiento actual sobre el endurecimiento rápido del hormigón. Parte el autor de los principios básicos y llega a las últimas consecuencias y realidades técnicas y econo micas.

Es una obra de consulta, tanto para el investigador sobre la materia, como para el proyectista y el realizador y montador de plantas e instalaciones y equipos de curado plantas e instalaciones y
y endurecimiento rápido.

Un volumen encuadernado en cartóné, de $17 \times 24,5 \mathrm{~cm}$, compuesto de 385 págs. 110 figuras y 10 tablas.

Precios: 2.500 ptas.; \$USA 36.00 .

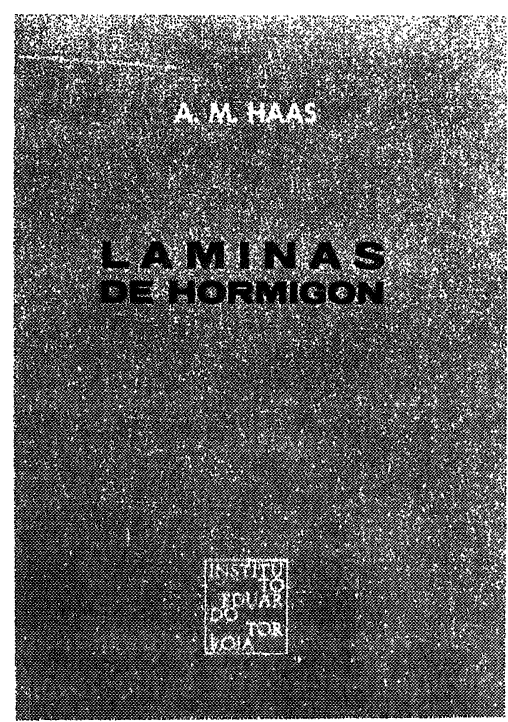

\section{A. M. Haas}

Al escribir este libro el autor intento pone a disposición de los estudiantes y de los ingenieros unos conocimientos prácticos, adecuados para servir de guia en el diseño y construcción de láminas delgadas de hormigón.

El autor está convencido de que el éxito en el diseño de una lámina exige, por parte de proyectista, un examen de las tres fases por las que pasa la materialización de la lámina: el diseño, el análisis estructural y la consel diseño, el análisis estruc
trucción de la estructura.

Un volumen encuadernado en tela, de $17 \times 24,5 \mathrm{~cm}$, compuesto de 420 págs., 141 figuras, 22 fotografias y 6 tablas.

Precios: 2.500 ptas.; SUSA 36.00 . 